\title{
ASYMPTOTIC SHAPE IN A CONTINUUM GROWTH MODEL
}

\author{
Maria Deijfen *
}

November 2002

\begin{abstract}
A continuum growth model is introduced. The state at time $t, S_{t}$, is a subset of $\mathbb{R}^{d}$ and consists of a connected union of randomly sized Euclidean balls, which emerge from outbursts at their center points. An outburst occurs somewhere in $S_{t}$ after an exponentially distributed time with expected value $\left|S_{t}\right|^{-1}$ and the location of the outburst is uniformly distributed over $S_{t}$. The main result is that if the distribution of the radii of the outburst balls has bounded support, then $S_{t}$ grows linearly and $S_{t} / t$ has a non-random shape as $t \rightarrow \infty$. Due to rotation invariance the asymptotic shape must be a Euclidean ball.
\end{abstract}

Keywords: First passage percolation; Richardson's model; subadditivity; shape theorem

AMS 2000 Subject Classification: Primary 60K35

Secondary 82B43.

\section{Introduction}

There is a variety of random growth models defined in a discrete space; see for instance Eden (1961), Williams and Bjerknes (1972), Bramson and Griffeath (1981) and Lee and Cowan (1994). A particular one is the Richardson model, introduced in Richardson (1973). It describes a Markov process whose state at time $t, S_{t}$, is a subset of $\mathbb{Z}^{d}$. Each site in $\mathbb{Z}^{d}$ is in either of two states, denoted 0 and 1 , and $S_{t}$ consists of the sites which are in state 1 at time $t$. A site in state 0 is transferred to state 1 at a rate proportional to the number of nearest neighbors in state 1 , and once in state 1 it never returns to state 0 . Thus, if sites in state 1 are thought of as infected sites and sites in state 0 as uninfected this dynamics defines a pure growth model. The main result, first proved in Richardson (1973), states that, if $S_{0}$ consists of a single site, then $S_{t} / t$ has a

*Postal address: Department of Mathematics, Stockholm University, SE-106 91 Stockholm, Sweden. E-mail: mia@matematik.su.se. 
non-random shape as $t \rightarrow \infty$. Generalizations of Richardson's result can be found in Cox and Durrett (1981), Kesten (1986) and Boivin (1990). Apart from the fact that the asymptotic shape is convex and compact, not much is known about its qualitative features.

In this paper we introduce a growth model defined in a continuous space, that is, the state at time $t$, still denoted by $S_{t}$, is a subset of $\mathbb{R}^{d}$ instead of $\mathbb{Z}^{d}$. The process should be thought of as describing the spread of some kind of infection in a continuous medium and just as in the Richardson model, the set $S_{t}$ specifies the region infected at time $t$. The growth takes place by way of outbursts in the infected region. Given the development of the infection up to time $t$, the time until an outburst occurs somewhere in $S_{t}$ is exponentially distributed with parameter $\left|S_{t}\right|$ and the location of the outburst is uniformly distributed over $S_{t}$ (the notation $|\cdot|$ will, throughout the paper, be used to denote both Lebesgue measure and Euclidean norm, but the meaning will always be obvious from the context). When an outburst occurs at an infected point it causes a ball of stochastic radius around the outburst point to be infected and the total infected region is enlarged by the amount of this ball that was not previously infected. Consequently the infected region is a connected union of Euclidean balls. The radii of these balls are assumed to be i.i.d. with distribution $F$ and mean $\gamma$.

The main result in this paper is a shape theorem for the continuum model. An essential advantage of the continuum model as compared to the Richardson model is that it possesses rotational invariance, which forces the asymptotic shape to be a Euclidean ball. To formulate the theorem, let $B(x, r)$ denote a ball with radius $r$ around the point $x \in \mathbb{R}^{d}$ and let $S_{t}$ denote the infected region at time $t$ in the $d$-dimensional continuum model starting at time zero from a ball with radius $\gamma$ around the origin.

Theorem 1.1 (Shape theorem) Assume that $F$ has bounded support and let $S_{0}=B(0, \gamma)$. Then, for any dimension $d$, there is a real number $\mu>0$ such that, for any $\varepsilon$ with $0<\varepsilon<\mu^{-1}$, almost surely

$$
(1-\varepsilon) B\left(0, \mu^{-1}\right) \subset \frac{S_{t}}{t} \subset(1+\varepsilon) B\left(0, \mu^{-1}\right)
$$

for all sufficiently large $t$.

Another example of a continuum model with a Euclidean ball as asymptotic shape is described in Howard and Newman (1997).

The condition that the support of $F$ is bounded can probably be weakened but some assumption on the tail is certainly necessary to ensure that $\mu>0$, i.e. to ensure that the growth is not faster than linear.

A natural question is how the asymptotic shape is affected if the region infected at time zero is chosen to be something other than a ball with radius $\gamma$ around the origin. The answer is given in the following theorem. 
Theorem 1.2 The conclusion of the shape theorem holds for any bounded initial set $S_{0}$ with strictly positive Lebesgue measure. Also, the number $\mu$ is independent of the initial set.

The rest of the paper is organized as follows. We start by describing the model more thoroughly in Section 2. In Section 3 we define some important quantities and prove a technical lemma. Section 4 contains results concerning the growth in a fixed direction. To obtain these results we formulate and prove a sharpened version of Liggett's (1985) subadditive ergodic theorem (Theorem 4.2). Section 5 is devoted to the proof of the shape theorem and the proof of Theorem 1.2 .

\section{Description of the model}

In this section we construct the model more formally by defining a Markov process whose state at time $t, S_{t}$, is a subset of $\mathbb{R}^{d}$. The process may be thought of as describing the spread of an infection (with no recoveries) or the growth of a germ colony in a continuous medium. Points in $S_{t}$ will be referred to as infected.

To define the model, let $N$ be a Poisson process on $\mathbb{R}^{d+1}$. The extra dimension represents the time dimension and the points of $N$ are hence denoted $\left(X_{k}, T_{k}\right)$, where $X_{k} \in \mathbb{R}^{d}$ and the last coordinate $T_{k}$ gives the location on the time axis. To each point in the Poisson process we associate a random radius $R_{k}$. The variables $\left\{R_{k}\right\}$ are assumed to be i.i.d. with mean $\gamma$. At time zero a ball with radius $\gamma$ around the origin, denoted by $B_{0}$, is infected. The idea now is to follow the cylinder $B_{0} \times \mathbb{R}$ upwards along the time axis until a point in the Poisson process is found. An outburst then takes place at this point generating a new infection ball $B_{1}$, whose size is given by the radius associated with this particular Poisson point. The new infected region is given by $B_{0} \cup B_{1}$. Scanning within the cylinder $\left(B_{0} \cup B_{1}\right) \times \mathbb{R}$ further upwards along the time axis we eventually hit a new Poisson point representing a new outburst and corresponding enlargement of the infected region. And so on.

To make this description more formal, for $S \subset \mathbb{R}^{d}$, let $N_{S \times \mathbb{R}}$ denote the restriction of $N$ to $S \times \mathbb{R}$. The growth of the infected area takes place at time points $\left\{T_{n}\right\}$ by aid of outbursts with radii $\left\{R_{n}\right\}$ at points $\left\{X_{n}\right\}$ obtained from the following recursion:

1. Let $X_{0}=0, T_{0}=0, R_{0}=\gamma$ and define $B_{n}=\left\{y \in \mathbb{R}^{d} ;\left|X_{n}-y\right| \leq R_{n}\right\}$, that is, $B_{n}$ is a ball in $\mathbb{R}^{d}$ with radius $R_{n}$ centered at $X_{n}$.

2. Given $\left\{X_{i} ; i \leq n\right\},\left\{T_{i} ; i \leq n\right\}$ and $\left\{R_{i} ; i \leq n\right\}$, the time $T_{n+1}$ is defined as

$$
T_{n+1}=\inf _{k}\left\{T_{k} ; T_{k}>T_{n} \text { and }\left(X_{k}, T_{k}\right) \in N_{\cup_{i=0}^{n} B_{i} \times \mathbb{R}}\right\}
$$

and $X_{n+1}$ is the (a.s. unique) point in $\mathbb{R}^{d}$ such that $\left(X_{n+1}, T_{n+1}\right) \in$ $N_{\cup i=0}^{n} B_{i} \times \mathbb{R}$. The radius $R_{n+1}$ is given by the radius associated with $\left(X_{n+1}, T_{n+1}\right)$. 
From the sequence $\left\{X_{n}\right\}$ a new sequence $\left\{S_{(n)}\right\}$ is constructed by defining $S_{(n)}=$ $\cup_{i=0}^{n} B_{i}$. The infected region at time $t$ is now given by

$$
S_{t}=S_{(n)} \quad \text { for } t \in\left[T_{n}, T_{n+1}\right) .
$$

Let us introduce the notation $\Delta_{n}=T_{n}-T_{n-1}, n \geq 1$, for the successive times between the outbursts. By construction of the model and properties of the Poisson process

$$
\Delta_{n+1} \mid \mathcal{F}_{n} \sim \operatorname{Exp}\left(\left|S_{(n)}\right|\right),
$$

where $\mathcal{F}_{n}=\sigma\left(X_{0}, \ldots, X_{n}, T_{0}, \ldots, T_{n}, R_{0}, \ldots, R_{n}\right)$. Given $S_{t}$, the memoryless property of the exponential distribution implies that the time until an outburst occurs somewhere in $S_{t}$ is exponentially distributed with parameter $\left|S_{t}\right|$. The location of the outburst, $X_{n}$ - where $n$ is such that $t \in\left[T_{n-1}, T_{n}\right)$ - is uniformly distributed over $S_{t}$ and the new infected region is given by $S_{t} \cup B_{n}$. Furthermore, the model is Markovian.

To guarantee that the model is defined for all $t$ one detail remains to be checked: We have to make sure that the sequence $\left\{T_{n}\right\}$ does not have a finite limit point $T_{\infty}$, since this would cause problems defining $S_{t}$ for $t>T_{\infty}$. The following proposition is what we need:

Proposition 2.1 Assume that the radii distribution has finite moment of order $d$. Then, almost surely, $T_{n} \rightarrow \infty$ as $n \rightarrow \infty$.

Proof: Let $\left\{E_{k}\right\}$ be independent, $E_{k} \sim \operatorname{Exp}(k)$. It is left to the reader to show that it suffices to prove that $\sum_{k=1}^{\infty} E_{k}=\infty$ with probability 1 . To establish this, introduce $\tilde{E}_{k}=E_{k}-\mathrm{E}\left[E_{k}\right]=E_{k}-1 / k$. Using the fact that $\sum_{k=1}^{\infty} \mathrm{E}\left[\tilde{E}_{k}^{2}\right]=\sum_{k=1}^{\infty} 1 / k^{2}<\infty$, Kolmogorov's three-series theorem implies that $\sum_{k=1}^{\infty} \tilde{E}_{k}$ converges almost surely. Thus

$$
\sum_{k=1}^{\infty} E_{k}=\sum_{k=1}^{\infty} \tilde{E}_{k}+\sum_{k=1}^{\infty} \frac{1}{k}=\infty
$$

since $\sum_{k=1}^{\infty} 1 / k=\infty$.

\section{Preliminaries}

In this section we introduce some preliminary notation and results needed to prove the shape theorem.

To begin with, let $T(x)$ denote the time when the point $x$ is infected, i.e.

$$
T(x)=\inf \left\{t ; x \in S_{t}\right\} .
$$

Our first result is a lemma bounding the time it takes for the infection to reach the point $x$. Here, for $z \in \mathbb{R},\lceil z\rceil$ is the smallest integer larger than $z$. 


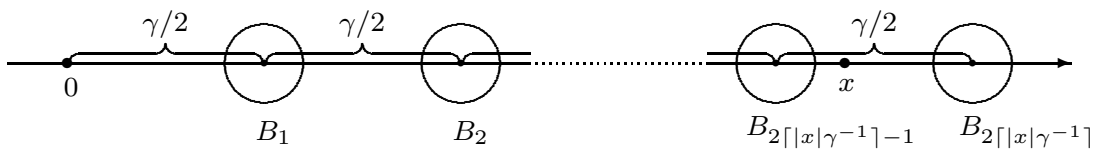

Figure 1: A chain of small balls located $\gamma / 2$ units apart on the line segment joining the origin and $x$ is constructed.

Lemma 3.1 For any $x \in \mathbb{R}^{d}$ there exist i.i.d. exponential variables $\left\{E_{k}\right\}$ with parameter $\lambda=\lambda(d)$ such that

$$
T(x) \leq \sum_{k=1}^{2\left\lceil|x| \gamma^{-1}\right\rceil} E_{k} .
$$

Proof: (We give the proof for $d=2$. The case $d \geq 3$ is analogous.)

Fix $x \in \mathbb{R}^{2}$ and assume, without loss of generality, that $x$ is located on the $x$-axis, that is, assume $x=\left(x^{\prime}, 0\right)$.

To begin with, fix $c \in \mathbb{R}$ and write $B_{k}$ for the ball of radius $c$ around the point $(k \cdot \gamma / 2,0)$, see Figure 1 . If $c$ is sufficiently small, say $c \leq \gamma / 10$, then $B_{1}$ is contained in $B(0, \gamma)$, that is, $B_{1}$ is infected at time zero. Let $E_{1}$ denote the time from time zero until an outburst with radius at least $\gamma$ occurs in $B_{1}$. Clearly $B_{2}$ is infected by the time such an outburst has occurred, that is, $B_{2} \subset S_{E_{1}}$. Now, the outburst points whose radius exceeds $\gamma$ constitutes a Poisson process with intensity $p=P\left(R_{k} \geq \gamma\right)$ and thus $E_{1} \sim \operatorname{Exp}\left(p \pi c^{2}\right)$, where $\pi c^{2}$ is the area of $B_{1}$.

The idea of how to continue should be clear: Let $E_{0}=0$ and define $E_{k}, k \geq 1$, recursively as the time until an outburst with radius larger than $\gamma$ occurs in $B_{k}$ counting from time $E_{0}+\ldots+E_{k-1}$. Since $B_{k} \subset S_{E_{1}+\ldots+E_{k-1}}$, we have that $E_{k} \sim \operatorname{Exp}\left(p \pi c^{2}\right)$. When an outburst has occurred in $B_{2\left\lceil|x| \gamma^{-1}\right\rceil}$ it is clear that $x$ must be infected, i.e. $x \in S_{E_{1}+\ldots+E_{2\left\lceil|x| \gamma^{-1}\right\rceil}}$. Hence

$$
T(x) \leq \sum_{k=1}^{2\left\lceil|x| \gamma^{-1}\right\rceil} E_{k}
$$

and the lemma is proved.

Remark 3.1 It follows from the proof of the lemma that the bound for $T(x)$ is valid also for the time until a small cube around $x$ is infected. By construction of the variables $\left\{E_{k}\right\}$, at time $\sum_{k=1}^{2\left\lceil|x| \gamma^{-1}\right\rceil} E_{k}$ an outburst with radius at least $\gamma$ has occurred somewhere in a ball with radius $c / 2, c \leq \gamma / 10$, centered at a point within distance $\gamma / 2$ from $x$. Thus, a cube centered at $x$ will be contained in the 
infected area at time $\sum_{k=1}^{2\left\lceil|x| \gamma^{-1}\right\rceil} E_{k}$ if the side length of the cube is chosen small enough. This observation will be useful in proving Lemma 5.1.

We now introduce some auxiliary quantities that will be of use later. Let $S_{t}^{(x, s)}$, $t \geq s$, denote the set of points that can be reached from $x$ within time $t$ if a new process is started at $x$ at time $s$. That is, at time $s$ all infection except a ball with radius $\gamma$ around $x$ is erased. The infection then evolves in time according to the same rules as for the original process, using the same $(d+1)$-dimensional Poisson process. This gives rise to a new process, emanating from $x$, whose state at time $t, t \geq s$, is given by $S_{t}^{(x, s)}$. Now let

$$
\tilde{T}(x)=\inf \left\{t ; B(x, \gamma) \subset S_{t}\right\}
$$

and

$$
\tilde{T}(x, y)=\inf \left\{t ; B(y, \gamma) \subset S_{\tilde{T}(x)+t}^{(x, \tilde{T}(x))}\right\} .
$$

In words, $\tilde{T}(x)$ is the time when the entire ball with radius $\gamma$ around $x$ is infected and $\tilde{T}(x, y)$ is the time it takes for the infection to invade the entire $\gamma$-ball around $y$ if a new process is started at $x$ at time $\tilde{T}(x)$. Note that the quantity $\tilde{T}(x, y)$ is independent of $\tilde{T}(x)$ and has the same distribution as $\tilde{T}(y-x)$. Furthermore, it is clear that, if a point is contained in the region infected at time $\tilde{T}(x)+t$ in the process started at $x$ at time $\tilde{T}(x)$, then it is also contained in the region infected at time $\tilde{T}(x)+t$ in the original process, i.e.

$$
S_{\tilde{T}(x)+t}^{(x, \tilde{T}(x))} \subset S_{\tilde{T}(x)+t}
$$

and hence

$$
\tilde{T}(y) \leq \tilde{T}(x)+\tilde{T}(x, y) .
$$

We close this section by anticipating that in what follows it will be convenient to have special notation for the quantity $\tilde{T}(m x, n x)$. Thus, let

$$
\tilde{T}(m x, n x)=\tilde{T}_{m, n}(x) .
$$

Since $\tilde{T}(n x)=\tilde{T}_{0, n}(x)$, the subadditivity property (1) translates into

$$
\tilde{T}_{0, n}(x) \leq \tilde{T}_{0, m}(x)+\tilde{T}_{m, n}(x) .
$$

\section{Growth in a fixed direction}

The proof of the shape theorem basically consists of two parts:

1. Show that $S_{t}$ grows linearly in each fixed direction and that the asymptotic speed of the growth in each direction is an almost sure constant. By the rotational invariance of $\mathbb{R}^{d}$ and the model, this constant must be the same for all directions. 
2. Show that the linear growth of $S_{t}$ holds for all directions simultaneously.

This section is devoted to the first part. The first task is to prove the following result.

Proposition 4.1 For each $x \in \mathbb{R}^{d}$, we have

(a) $\mu(x):=\lim _{n \rightarrow \infty} \mathrm{E}[\tilde{T}(n x)] / n=\inf _{n \geq 1} \mathrm{E}[\tilde{T}(n x)] / n$;

(b) $\lim _{n \rightarrow \infty} \tilde{T}(n x) / n=\mu(x)$ a.s.

Remark 4.1 We employ the convention that limits over $n$ are taken over the positive integers, while limits over $t$, which occur later on in the paper, are taken over all positive reals.

To prove Proposition 4.1 we will invoke the following theorem by Liggett (1985).

Theorem 4.1 (Liggett's subadditive ergodic theorem) Let $\left\{X_{m, n}\right\}$ be a collection of random variables indexed by integers satisfying $0 \leq m<n$. Suppose $\left\{X_{m, n}\right\}$ has the following properties:

(i) $X_{0, n} \leq X_{0, m}+X_{m, n}$.

(ii) For each $n, \mathrm{E}\left|X_{0, n}\right|<\infty$ and $\mathrm{E}\left[X_{0, n}\right] \geq$ cn for some constant $c>-\infty$.

(iii) The distribution of $\left\{X_{m, m+k} ; k \geq 1\right\}$ does not depend on $m$.

(iv) For each $k \geq 1,\left\{X_{n k,(n+1) k} ; n \geq 0\right\}$ is a stationary sequence.

Then

(a) $\eta:=\lim _{n \rightarrow \infty} \mathrm{E}\left[X_{0, n}\right] / n=\inf _{n \geq 1} \mathrm{E}\left[X_{0, n}\right] / n$.

(b) The limit $X:=\lim _{n \rightarrow \infty} X_{0, n} / n$ exists a.s.

(c) $\mathrm{E}[X]=\eta$.

Furthermore, if the stationary processes in (iv) are ergodic, then

(d) $X=\eta$ a.s.

A brief outline of the structure of the proof can be found below. For more detail we refer the reader to Liggett (1985).

We wish to apply this result to the variables $\left\{\tilde{T}_{m, n}(x)\right\}$. It turns out, however, that condition (iv) fails for the sequence $\left\{\tilde{T}_{n k,(n+1) k}(x) ; n \geq 0\right\}$. Luckily the assumption (iv) can be relaxed without weakening the conclusions:

Theorem 4.2 Let $\left\{X_{m, n}\right\}$ be a collection of random variables satisfying(i)-(iii) of Theorem 4.1. Furthermore, suppose that

(iv') $\lim \sup _{n \rightarrow \infty} X_{0, n k} / n \leq \mathrm{E}\left[X_{0, k}\right]$ for each $k$. 
Then (a)-(d) of Theorem 4.1 hold for $\left\{X_{m, n}\right\}$.

Remark 4.2 A trivial modification of the proof of Theorem 4.2 yields that, if we confine ourselves with the conclusions (a)-(c), then (iv') can be replaced by the still weaker assumption

(iv") $\mathrm{E}\left[\lim \sup _{n \rightarrow \infty} X_{0, n k} / n\right] \leq \mathrm{E}\left[X_{0, k}\right]$ for each $k$.

To prove Theorem 4.2 requires some knowledge of the structure of the proof of Theorem 4.1. This knowledge is provided in the following brief sketch.

Write

$$
\bar{X}=\limsup _{n \rightarrow \infty} \frac{X_{0, n}}{n}
$$

and

$$
X=\liminf _{n \rightarrow \infty} \frac{X_{0, n}}{n} .
$$

The proof of Theorem 4.1 is broken up into three steps:

(L1) Prove that $\eta=\lim _{n \rightarrow \infty} \mathrm{E}\left[X_{0, n}\right] / n=\inf _{n \geq 1} \mathrm{E}\left[X_{0, n}\right] / n$.

(L2) Prove that $\mathrm{E}[\bar{X}] \leq \eta$, and if the stationary processes in (iv) are ergodic, then $\bar{X} \leq \eta$ almost surely.

(L3) Prove that $\mathrm{E}[\underline{X}] \geq \eta$.

From (L2) and (L3) it follows that $\mathrm{E}[\underline{X}] \geq \mathrm{E}[\bar{X}]$. This implies that $X$ and $\bar{X}$ are equal, since trivially $X \leq \bar{X}$. Hence, once (L2) and (L3) are accomplished it is clear that $X:=\lim _{n \rightarrow \infty} X_{0, n} / n$ exists with probability 1 . It also follows from (L2) and (L3) that $\mathrm{E}[X]=\eta$ and by (L1) that $\eta<\infty$. Furthermore, if $\bar{X} \leq \eta$-which, according to (L2), for example is the case if the sequences $\left\{X_{n k,(n+1) k} ; n \geq 0\right\}$ are ergodic - then we can deduce that $X=\eta$ almost surely.

For (L1)-(L3) we refer the reader to Liggett (1985). The essential task for us is to identify the parts of the proof that make use of the assumption (iv). Since this assumption is to be replaced by (iv'), these are the parts that have to be modified. The following table shows how the assumptions are used in the different steps.

\begin{tabular}{c|c} 
Step & Assumptions used \\
\hline (L1) & (i), (iii) \\
(L2) & (i)-(iv) \\
(L3) & (i)-(iii)
\end{tabular}

Since the proofs of (L1) and (L3) do not use (iv), these statements hold also if (iv) is dropped. In proving (L2) though, Liggett uses the assumption (iv) and thus a modification of Liggett's proof is necessary to establish that (L2) 
remains true when (iv) is replaced by (iv'). The modification is described in the following proof of Theorem 4.2 .

Proof of Theorem 4.2: It suffices to show that $\bar{X} \leq \eta$ almost surely, that is, it suffices to show that

$$
\limsup _{n \rightarrow \infty} \frac{X_{0, n}}{n} \leq \eta \quad \text { a.s. }
$$

To achieve this, fix $\delta>0$ and choose $k$ large so that $\mathrm{E}\left[X_{0, k}\right] / k \leq \eta+\delta$. We will show that for all $j$,

$$
\limsup _{n \rightarrow \infty} \frac{X_{0, n k+j}}{n k+j} \leq \frac{\mathrm{E}\left[X_{0, k}\right]}{k} \quad \text { a.s. }
$$

which yields

$$
\limsup _{n \rightarrow \infty} \frac{X_{0, n}}{n} \leq \frac{\mathrm{E}\left[X_{0, k}\right]}{k} \text { a.s. }
$$

Hence, once (4) has been established it follows from the choice of $k$ that

$$
\limsup _{n \rightarrow \infty} \frac{X_{0, n}}{n} \leq \eta+\delta
$$

and, since $\delta>0$ was arbitrary, this implies (3). To prove (4), fix $j$ and use subadditivity to get

$$
\frac{X_{0, n k+j}}{n k+j} \leq \frac{n}{n k+j} \cdot \frac{X_{0, n k}}{n}+\frac{n}{n k+j} \cdot \frac{X_{n k, n k+j}}{n} .
$$

By (iii), the distribution of $X_{n k, n k+j}$ depends only on $j$ and, by (ii), the first moment is finite. Thus,

$$
\sum_{n=1}^{\infty} P\left(X_{n k, n k+j}>n \varepsilon\right)<\infty
$$

for all $\varepsilon>0$ and by the Borel-Cantelli lemma this implies that

$$
\lim _{n \rightarrow \infty} \frac{X_{n k, n k+j}}{n}=0 \quad \text { a.s. }
$$

Using (iv'), (4) follows from (5) and (6).

We are now in a position to prove Proposition 4.1.

Proof of Proposition 4.1: Fix $x \in \mathbb{R}^{d}$. Since $\left\{\tilde{T}_{m, n}(x)\right\}$ satisfies the assumptions (i)-(iii) of Theorem 4.1, the proposition follows from Theorem 4.2 if we can show that (iv') holds for $\left\{\tilde{T}_{m, n}(x)\right\}$, that is, if we can show that

$$
\limsup _{n \rightarrow \infty} \frac{\tilde{T}_{0, n k}(x)}{n} \leq \mathrm{E}\left[T_{0, k}\right] \text { for all } k .
$$

To do this it is necessary to introduce an auxiliary sequence $\left\{\tilde{T}_{(i-1) k, i k}^{\prime}(x)\right.$; $i \geq 1\}$ defined recursively as follows: 
Let $\tilde{T}_{0, k}^{\prime}(x)=\tilde{T}_{0, k}(x)$. For $i \geq 2$, given $\left\{\tilde{T}_{(l-1) k, l k}^{\prime}(x) ; l \leq i-1\right\}$, define

$$
\tilde{T}_{(i-1) k, i k}^{\prime}(x)=\inf \left\{t ; B(i k x, \gamma) \subset S_{\Phi_{i-1}^{k}+t}^{\left((i-1) k x, \Phi_{i-1}^{k}\right)}\right\},
$$

where $\Phi_{i-1}^{k}=\sum_{l=1}^{i-1} \tilde{T}_{(l-1) k, l k}^{\prime}(x)$.

Remember that $S_{t}^{(x, s)}$ is the area infected at time $t, t \geq s$, in a process started at time $s$ emanating from a ball with radius $\gamma$ around $x$. Thus, $\tilde{T}_{(i-1) k, i k}^{\prime}(x)$ is the time when the ball with radius $\gamma$ around $i k x$ is infected in a process started at $(i-1) k x$ at time $\Phi_{i-1}^{k}$. Some thought reveals that the variables $\left\{\tilde{T}_{(i-1) k, i k}^{\prime}(x)\right\}$ are i.i.d. with expected value $E\left[\tilde{T}_{0, k}(x)\right]$. Hence, by the strong law of large numbers,

$$
\frac{1}{n} \sum_{i=1}^{n} \tilde{T}_{(i-1) k, i k}^{\prime}(x) \rightarrow \mathrm{E}\left[\tilde{T}_{0, k}(x)\right] \quad \text { as } n \rightarrow \infty .
$$

Furthermore, it is readily seen that

$$
\tilde{T}_{0, n k}(x) \leq \sum_{i=1}^{n} \tilde{T}_{(i-1) k, i k}^{\prime}(x) .
$$

Dividing this inequality by $n$ and using (8) we obtain (7). Theorem 4.2 now gives that all the conclusions (a)-(d) of Theorem 4.1 hold for $\left\{\tilde{T}_{m, n}(x)\right\}$. Part (a) of the proposition follows from 4.1(a) and part (b) follows from Theorem 4.1(b) and (d).

Our next task is to show that $\mu(x)$ is nonzero so that the growth is indeed linear.

Proposition 4.2 If $F$ has bounded support, then $0<\mu(x)<\infty$ for each $x \in$ $\mathbb{R}^{d}, x \neq 0$.

Proof: Fix $x \in \mathbb{R}^{d}$. That $\mu(x)<\infty$ follows from Proposition4.1(a) so it remains to show that $\mu(x)>0$. Since $F$ has bounded support, there is a real number $r_{\max }$ such that with probability 1 the radius of an outburst ball does not exceed $r_{\max }$. Using this, it follows from a minor modification of Lemma 3.1 in Penrose $(2001) \sqrt{1}$ that there are constants $c_{1}, c_{2} \in(0, \infty)$ such that

$$
P\left(T(x) \leq c_{1}|x|\right) \leq 2 \cdot 3^{-c_{2}|x|} .
$$

That $\mu(x)$ is nonzero follows from (9) and the fact that

$$
\limsup _{n \rightarrow \infty} \frac{\mathrm{E}[T(n x)]}{n} \leq \lim _{n \rightarrow \infty} \frac{\mathrm{E}[\tilde{T}(n x)]}{n}=\mu(x)
$$

\footnotetext{
${ }^{1}$ or see the appendix on p. 47.
} 
by arguing as follows: Substituting $x$ by $n x$ in (9) yields

$$
P\left(\frac{T(n x)}{n} \leq c_{1}|x|\right) \leq 2 \cdot 3^{-c_{2} n|x|},
$$

which implies that

$$
\frac{\mathrm{E}[T(n x)]}{n} \geq c_{1}|x|\left(1-2 \cdot 3^{-c_{2} n|x|}\right) .
$$

Using (10) this gives that $\mu(x) \geq c_{1}|x|$.

The next step is to prove that the discrete limit in Proposition 4.1(b) can be replaced by a continuous one.

Proposition 4.3 For each $x \in \mathbb{R}^{d}$ we have $\lim _{t \rightarrow \infty} \tilde{T}(t x) / t=\mu(x)$, where the limit is taken along $t \in \mathbb{R}^{+}$.

Proof: Fix $x \in \mathbb{R}^{d}$. We start by showing that

$$
\lim _{n \rightarrow \infty} \frac{\tilde{T}(n q x)}{n q}=\mu(x) \quad \text { for all } q \in \mathbb{Q}
$$

that is, moving away from the origin in direction $x$ using steps of arbitrary rational length yields the same limit. To this end, introduce the notation

$$
\bar{T}_{t}=\frac{\tilde{T}(t x)}{t}
$$

Now, since $q \in \mathbb{Q}$ we have $q=k / m$ for some integers $k$ and $m$. The sequence $\left\{\bar{T}_{n k}\right\}$ is a subsequence of $\left\{\bar{T}_{n}\right\}$ and, hence, by Proposition 4.1(b),

$$
\lim _{n \rightarrow \infty} \bar{T}_{n k}=\lim _{n \rightarrow \infty} \bar{T}_{n}=\mu(x) \quad \text { a.s. }
$$

However, $\left\{\bar{T}_{n k}\right\}$ is also a subsequence of $\left\{\bar{T}_{n q}\right\}$ - obtained by considering only those points where $n$ is a multiple of $m$ - and since the sequence $\left\{\bar{T}_{n q}\right\}$ does indeed have a limit (this follows from Proposition 4.1(b) applied to the point $q x)$ this implies that

$$
\lim _{n \rightarrow \infty} \bar{T}_{n q}=\lim _{n \rightarrow \infty} \bar{T}_{n k}=\mu(x) \quad \text { a.s. }
$$

To complete the proof we use (11) to show that

$$
\limsup _{t \rightarrow \infty} \frac{\tilde{T}(t x)}{t} \leq \mu(x) \leq \liminf _{t \rightarrow \infty} \frac{\tilde{T}(t x)}{t} .
$$

To establish (12) we will need a bound for the time from the moment when the $\gamma$-ball around an arbitrary point on the line segment between $n q x$ and $(n+1) q x$ is infected until the $\gamma$-balls of all points on the line segment are infected. To obtain such a bound, let $l_{n q x}$ denote the line segment between $n q x$ and $(n+1) q x$ 


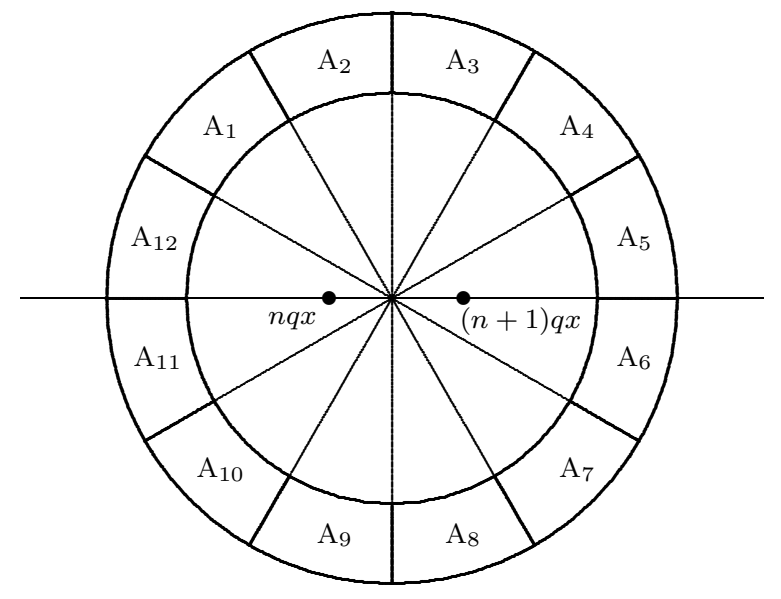

Figure 2: The front zone $F$ of $B\left(z_{0}, \gamma\right)$ divided into pieces $A_{1}, \ldots, A_{12}$.

and write $\tilde{T}\left(l_{n q x}\right)$ for the time when all points on $l_{n q x}$ has their $\gamma$-balls infected, i.e.

$$
\tilde{T}\left(l_{n q x}\right)=\inf \left\{t ; B(z, \gamma) \subset S_{t} \text { for all } z \in l_{n q x}\right\} .
$$

Assume that at time $t_{0}$ there is a point $z_{0} \in l_{n q x}$ such that $B\left(z_{0}, \gamma\right) \subset S_{t_{0}}$. For small $q \in \mathbb{Q}$ we will derive an upper bound for $\tilde{T}\left(l_{n q x}\right)-t_{0}$ expressed as the maximum of a number of i.i.d. exponential random variables. In two dimensions such a bound is easily obtained using a geometric construction displayed in Figure 2. Namely, let $F$ be the outer ring of width $\gamma / 10$ in $B\left(z_{0}, \gamma\right)$, that is, $F=B\left(z_{0}, \gamma\right) \backslash B\left(z_{0}, 9 \gamma / 10\right)$. Divide $F$ into twelve disjoint pieces $A_{1}, \ldots, A_{12}$ of equal area as shown in Figure 2 and let $E_{k}(k=1, \ldots, 12)$ be the time from time $t_{0}$ until an outburst with radius at least $\gamma$ occurs in $A_{k}$. By construction, the areas $A_{1}, \ldots, A_{12}$ are all infected at time $t_{0}$, implying that $E_{k} \sim \operatorname{Exp}\left(p\left|A_{k}\right|\right)$. Furthermore, since the $A_{k}$ are disjoint, the variables $\left\{E_{k}\right\}$ are independent. Now, if $q$ is small, then all points on $l_{n q x}$ must have their $\gamma$-balls infected by the time an outburst whose radius exceeds $\gamma$ has occurred in each of the areas $A_{k}(k=1, \ldots, 12)$. Hence $B(z, \gamma) \subset S_{t_{0}+\max \left\{E_{1}, \ldots, E_{12}\right\}}$ for all $z \in l_{n q x}$ and we have proved that $\tilde{T}\left(l_{n q x}\right)-t_{0} \leq \max \left\{E_{1}, \ldots, E_{12}\right\}$ for all times $t_{0}$ such that there exists a point on $l_{n q x}$ whose entire $\gamma$-ball is infected at time $t_{0}$. The above reasoning easily generalizes to $d \geq 3$. We obtain

$$
\tilde{T}\left(l_{n q x}\right)-t_{0} \leq \max \left\{E_{1}, \ldots, E_{k_{0}}\right\},
$$

where $k_{0}=k_{0}(d)$ and $E_{k} \sim \operatorname{Exp}(\lambda(d))$.

Now, to prove the first inequality in (12) let $q \in \mathbb{Q}$ be small enough to ensure (13) and let $\tilde{\varphi}_{n q}(x)$ be the time when the $\gamma$-balls of all points on $l_{n q x}$ are infected counting from the time when the $\gamma$-ball around $n q x$ is infected, that 
is, $\tilde{\varphi}_{n q}(x)=\tilde{T}\left(l_{n q x}\right)-\tilde{T}(n q x)$. By (13) there exist i.i.d. random variables $E_{1}, \ldots, E_{k_{0}}$ such that

$$
\tilde{\varphi}_{n q}(x) \leq \max \left\{E_{1}, \ldots, E_{k_{0}}\right\}:=M_{k_{0}} .
$$

Since $M_{k_{0}} \leq \sum_{k=1}^{k_{0}} E_{k}$, it follows that $\mathrm{E}\left[M_{k_{0}}\right] \leq k_{0} \mathrm{E}\left[E_{1}\right]<\infty$ and, hence, for all $\varepsilon>0$,

$$
\sum_{n=0}^{\infty} P\left(M_{k_{0}}>n \varepsilon\right)<\infty .
$$

Thus, by (14) and the Borel-Cantelli lemma,

$$
\lim _{n \rightarrow \infty} \frac{\tilde{\varphi}_{n q}(x)}{n}=0 \quad \text { a.s. }
$$

Let $n_{t}$ be such that $t \in\left[n_{t} q,\left(n_{t}+1\right) q\right)$. Clearly $\tilde{T}(t x) \leq \tilde{T}\left(n_{t} q x\right)+\tilde{\varphi}_{n q}(x)$. Hence

$$
\begin{aligned}
\limsup _{t \rightarrow \infty} \frac{\tilde{T}(t x)}{t} & \leq \limsup _{t \rightarrow \infty} \frac{\tilde{T}\left(n_{t} q x\right)+\tilde{\varphi}_{n_{t} q}(x)}{n_{t} q} \\
& =\lim _{n \rightarrow \infty} \frac{\tilde{T}(n q x)+\tilde{\varphi}_{n q}(x)}{n q} \\
& =\mu(x),
\end{aligned}
$$

where the last equality follows from (11) and (15).

To prove the last inequality in (12), let $z_{n q x}$ be the first point on $l_{n q x}$ whose $\gamma$-ball is infected and let $\tilde{\psi}_{n q}(x)$ be the time from when this occurs until the infection has invaded the $\gamma$-balls of all points on $l_{n q x}$, that is, $\tilde{\psi}_{n q}(x)=\tilde{T}\left(l_{n q x}\right)-\tilde{T}\left(z_{n q x}\right)$. Using (13) and Borel-Cantelli it can be seen that

$$
\lim _{n \rightarrow \infty} \frac{\tilde{\psi}_{n c}(x)}{n}=0 \quad \text { a.s. }
$$

Since $\tilde{T}(t x)+\tilde{\psi}_{n_{t} q}(x) \geq \tilde{T}\left(n_{t} q x\right)$, we have

$$
\begin{aligned}
\liminf _{t \rightarrow \infty} \frac{\tilde{T}(t x)}{t} & \geq \liminf _{t \rightarrow \infty} \frac{\tilde{T}\left(n_{t} q x\right)-\tilde{\psi}_{n_{t} q}(x)}{\left(n_{t}+1\right)} \\
& =\lim _{n \rightarrow \infty} \frac{\tilde{T}(n q x)-\tilde{\psi}_{n q}(x)}{(n+1) q} \\
& =\mu(x),
\end{aligned}
$$

where the last equality is a consequence of (11) and (17). Thereby (12) is established and the proposition follows. 
It follows from Proposition 4.3 that $\mu(c x)=c \mu(x)$. This implies that $\mu(x)=$ $|x| \mu(\hat{x})$, where $\hat{x}=x /|x|$, the unit vector in direction $x$. Due to rotational invariance of $\mathbb{R}^{d}$ and the model it is clear that $\mu(\hat{x})=\mu(\hat{y})$ for all $x, y \in \mathbb{R}^{d}$. Thus we can define a constant

$$
\mu:=\mu((1,0, \ldots, 0))
$$

representing the asymptotic time it takes for the infection to travel a unit vector in an arbitrary direction. By Proposition $4.2, \mu \in(0, \infty)$. We end up with the simple relation $\mu(x)=|x| \mu$ valid for all $x \in \mathbb{R}^{d}$.

To summarize the results obtained in the present section, we have deduced that there is a real number $\mu>0$ such that, for each fixed $x \in \mathbb{R}^{d}$, almost surely

$$
\lim _{t \rightarrow \infty} \frac{\tilde{T}(t x)}{t}=|x| \mu
$$

\section{Proof of the shape theorem}

The shape theorem asserts that $S_{t} \approx t B\left(0, \mu^{-1}\right)$ for large $t$ and in view of (19) this is indeed what to expect. However, it remains to show that the linear growth stipulated in (19) holds for all directions simultaneously. To this end we will need the following lemma, which asserts that with high probability the infected region in a process emanating from a point $y$ will eventually contain a ball centered at $y$ with radius proportional to time.

Lemma 5.1 For small $\delta>0$, there is a constant $c \in(0, \infty)$ and a time $s_{0}$ such that, for any $y \in \mathbb{R}^{d}$ and $s^{\prime} \geq 0$, we have

$$
P\left(B(y, s \delta) \not \subset S_{s^{\prime}+s}^{\left(y, s^{\prime}\right)}\right) \leq e^{-c s}
$$

if $s>s_{0}$.

Proof of Lemma 5.1: Due to shift invariance of the model it suffices to prove the lemma for $y=0$ and $s^{\prime}=0$, that is, it suffices to show that

$$
P\left(B(0, s \delta) \not \subset S_{s}\right) \leq e^{-c s}
$$

for large $s$. To achieve this, fix a real number $\alpha>0$ and partition $\mathbb{R}^{d}$ into cubes centered at the points $\alpha \mathbb{Z}^{d}$ and with vertices $(\alpha / 2, \ldots, \alpha / 2)+\alpha \mathbb{Z}^{d}$. Furthermore, let $\hat{T}_{\alpha}(x), x \in \alpha \mathbb{Z}^{d}$, denote the time when the entire cube centered at $x$ is infected. For small $\alpha$ and $\delta$ we will show that there is a positive constant $c^{\prime}$ such that the estimate

$$
P\left(\hat{T}_{\alpha}(x)>a|x| / \delta\right) \leq e^{-c^{\prime} a|x|}
$$

holds simultaneously for all $a \geq 1 / d$ and all $x \in \mathbb{Z}^{d}$. Given this estimate the lemma is readily established: Let $C(s \delta+\alpha)$ denote the cube with side length 
$2(s \delta+\alpha)$ centered at the origin and write $C_{\alpha}(s \delta+\alpha)=\alpha \mathbb{Z}^{d} \cap C(s \delta+\alpha)$. Note that $B(0, s \delta)$ is contained in the union of all $\alpha$-cubes whose centers are in $C_{\alpha}(s \delta+\alpha)$. Thus, if $B(0, s \delta) \not \subset S_{s}$, then not all $\alpha$-cubes with centers in $C_{\alpha}(s \delta+\alpha)$ are infected at time $s$, that is,

$$
\begin{aligned}
P\left(B(0, s \delta) \not \subset S_{s}\right) & \leq P\left(\bigcup_{x \in C_{\alpha}(s \delta+\alpha)}\left\{\hat{T}_{\alpha}(x)>s\right\}\right) \\
& \leq \sum_{x \in C_{\alpha}(s \delta+\alpha)} P\left(\hat{T}_{\alpha}(x)>s\right) .
\end{aligned}
$$

Trivially,

$$
P\left(\hat{T}_{\alpha}(x)>s\right)=P\left(\hat{T}_{\alpha}(x)>\frac{s \delta}{|x|} \cdot \frac{|x|}{\delta}\right) .
$$

For $x \in C_{\alpha}(s \delta+\alpha)$, we have $|x| \leq \sqrt{d}(s \delta+\alpha)$ and, since $s \delta / \sqrt{d}(s \delta+\alpha) \rightarrow 1 / \sqrt{d}$ as $s \rightarrow \infty$, it holds that $s \delta /|x| \geq 1 / d$ for large $s$. Hence, if $s$ is large and $x \in C_{\alpha}(s \delta+\alpha)$ it follows from (21) that

$$
P\left(\hat{T}_{\alpha}(x)>\frac{s \delta}{|x|} \cdot \frac{|x|}{\delta}\right) \leq e^{-c^{\prime} s \delta}
$$

and, consequently, for large $s$,

$$
P\left(B(0, s \delta) \not \subset S_{s}\right) \leq \sum_{x \in C_{\alpha}(s \delta+\alpha)} e^{-c^{\prime} s \delta} .
$$

Since the number of points in $C_{\alpha}(s \delta+\alpha)$ grows only polynomially in $s$ there is a time $s_{0}$ such that, for $s>s_{0}$, we have $P\left(B(0, s \delta) \not \subset S_{s}\right) \leq e^{-s c^{\prime} \delta / 2}$ as desired.

It remains to prove (21). Fix $x \in \alpha \mathbb{Z}^{d}$. By Lemma 3.1 and Remark 3.1, if $\alpha$ is small, say $\alpha \leq \gamma / 10$, we have

$$
\hat{T}_{\alpha}(x) \leq \sum_{k=1}^{2\left\lceil|x| \gamma^{-1}\right\rceil} E_{k},
$$

where $\left\{E_{k}\right\}$ are i.i.d. exponential variables with parameter $\lambda$. Thus, it suffices to find $c^{\prime}>0$ such that

$$
P\left(\sum_{k=1}^{2\left\lceil|x| \gamma^{-1}\right\rceil} E_{k}>\frac{a|x|}{\delta}\right) \leq e^{-c^{\prime} a|x|} .
$$

To this end, write $\left\lceil|x| \gamma^{-1}\right\rceil=m$ and introduce the notation $\Gamma_{2 m}:=\sum_{k=1}^{2 m} E_{k}$. Using Markov's inequality and the fact that $\Gamma_{2 m} \sim \operatorname{Gamma}(2 m, \lambda)$, we obtain

$$
e^{\theta a|x| \delta^{-1}} P\left(\Gamma_{2 m}>a|x| \delta^{-1}\right) \leq \mathrm{E}\left[e^{\theta \Gamma_{2 m}}\right]=(1-\lambda \theta)^{-2 m}
$$


for $\theta \in\left(0, \lambda^{-1}\right)$. Thus

$$
P\left(\Gamma_{2 m}>a|x| \delta^{-1}\right) \leq \exp \left\{-a|x|\left(\theta \delta^{-1}+\frac{2 m}{a|x|} \log (1-\theta \lambda)\right)\right\}
$$

We may assume that $|x|>\gamma-\alpha$ since, for $x \in B(0, \gamma-\alpha)$, the $\alpha$-cube centered at $x$ is contained in $B(0, \gamma)$, implying that the left hand side in (21) equals zero and hence (21) is trivially true in this case. For $|x|>\gamma-\alpha$, the quotient $m /|x|=\left\lceil|x| \gamma^{-1}\right\rceil /|x|$ is bounded by $2 \gamma^{-1}$. Substituting this in (23) and also using the fact that $a \geq 1 / d$ yields

$$
P\left(\Gamma_{2 m}>a|x| \delta^{-1}\right) \leq e^{-a|x| f_{\delta}(\theta)},
$$

where $f_{\delta}(\theta)=\theta \delta^{-1}+4 d \gamma^{-1} \log (1-\lambda \theta)$. Now, $f_{\delta}(0)=0$ and $f_{\delta}^{\prime}(0)=\delta^{-1}-$ $4 d \gamma^{-1} \lambda$. Thus, if $\delta$ is so small that $f_{\delta}^{\prime}(0)>0$, then we can pick $\theta$ small and get $f_{\delta}(\theta)>0$. This proves (22).

Finally, equipped with the above lemma and the results from Section 4, we are ready to prove the shape theorem.

Proof of Theorem 1.1: Fix $\varepsilon \in\left(0, \mu^{-1}\right)$. We will prove the theorem in two steps:

(i) There is almost surely a time $T^{1}$ such that $(1-\varepsilon) t B\left(0, \mu^{-1}\right) \subset S_{t}$ for $t>T^{1}$.

(ii) There is almost surely a time $T^{2}$ such that $S_{t} \subset(1+\varepsilon) t B\left(0, \mu^{-1}\right)$ for $t>T^{2}$.

As for (i) we will, in fact, prove the following:

(i') There is almost surely a time $T^{1}$ such that $(1-\varepsilon / 2) t B\left(0, \mu^{-1}\right) \subset S_{t}$ for $t>T^{1}, t \in \mathbb{N}$.

From (i') it follows that $(1-\varepsilon / 2)\lfloor t\rfloor B\left(0, \mu^{-1}\right) \subset S_{\lfloor t\rfloor}$ for $t>T^{1}+1$, where $\lfloor\cdot\rfloor$ is the integer part function. Since $S_{\lfloor t\rfloor} \subset S_{t}$ for all $t$, we obtain (1$\varepsilon / 2)\lfloor t\rfloor B\left(0, \mu^{-1}\right) \subset S_{t}$. But, for large $t$, the ball with radius $(1-\varepsilon / 2)\lfloor t\rfloor \mu^{-1}$ contains the ball with radius $(1-\varepsilon) t \mu^{-1}$, and hence (i) follows from (i').

To prove (i'), note that, since $(1-\varepsilon / 2) B\left(0, \mu^{-1}\right)$ is compact, there are points $x_{1}, \ldots, x_{n} \in(1-\varepsilon / 2) B\left(0, \mu^{-1}\right)$ such that

$$
(1-\varepsilon / 2) B\left(0, \mu^{-1}\right) \subset \bigcup_{i=1}^{n} B\left(x_{i}, \delta \varepsilon / 4\right),
$$

where $\delta>0$ is chosen small enough to ensure that Lemma 5.1 holds. Clearly

$$
(1-\varepsilon / 2) t B\left(0, \mu^{-1}\right) \subset \bigcup_{i=1}^{n} B\left(t x_{i}, t \delta \varepsilon / 4\right) .
$$


Since $\left|x_{i}\right| \leq(1-\varepsilon / 2) \mu^{-1}$ it follows from (19) that, for each $i, \lim _{t \rightarrow \infty} \tilde{T}\left(t x_{i}\right) / t \leq$ $1-\varepsilon / 2$ almost surely. This implies that almost surely $\tilde{T}\left(t x_{i}\right) \leq t(1-\varepsilon / 4)$ for each $i$ if $t$ is large, that is,

$$
B\left(t x_{i}, \gamma\right) \subset S_{t(1-\varepsilon / 4)}
$$

for large $t$. Furthermore, by Lemma 5.1.

$$
\sum_{t \in \mathbb{N}} P\left(B\left(t x_{i}, t \delta \varepsilon / 4\right) \not \subset S_{t}^{\left(t x_{i}, t(1-\varepsilon / 4)\right)}\right) \sim \sum_{t \in \mathbb{N}} e^{-c t \varepsilon / 4}<\infty .
$$

Thus, by the Borel-Cantelli lemma, for large integer times we have almost surely

$$
B\left(t x_{i}, t \delta \varepsilon / 4\right) \subset S_{t}^{\left(t x_{i}, t(1-\varepsilon / 4)\right)} .
$$

Now, for each $i$, let $T(i)$ be such that both (25) and (26) hold for $t>T(i)$, $t \in \mathbb{N}$, and define $T^{1}=\max \{T(i)\}$. For $t>T^{1}$, we have $B\left(t x_{i}, t \delta \varepsilon / 4\right) \subset S_{t}$ for all $i$ and $t \in \mathbb{N}$. Using (24) this implies that $(1-\varepsilon / 2) t B\left(0, \mu^{-1}\right) \subset S_{t}$ for all integer times larger than $T_{1}^{\prime}$, as desired.

Moving on to (ii), let $A$ be an annulus of width $\mu^{-1} \varepsilon / 2$ surrounding $(1+\varepsilon / 2)$ $B\left(0, \mu^{-1}\right)$, i.e.

$$
A=(1+\varepsilon) B\left(0, \mu^{-1}\right) \backslash(1+\varepsilon / 2) B\left(0, \mu^{-1}\right),
$$

and let $\tilde{S}_{t}$ be the set of points whose entire $\gamma$-ball is infected at time $t$, i.e.

$$
\tilde{S}_{t}=\left\{x ; B(x, \gamma) \subset S_{t}\right\}
$$

Points in $\tilde{S}_{t}$ will be referred to as strongly infected at time $t$. We begin by showing that almost surely $\tilde{S}_{t} \cap t A=\emptyset$ for large $t$.

Let $\delta$ be small enough to ensure that Lemma 5.1 holds and pick $x_{1}, \ldots, x_{n} \in A$ such that

$$
A \subset \bigcup_{i=1}^{n} B\left(x_{i}, \delta \varepsilon / 8\right)
$$

By (19), $\lim _{t \rightarrow \infty} \tilde{T}\left(t x_{i}\right) / t \geq 1+\varepsilon / 2$ for each $i$. Hence, for each $i$, almost surely $\tilde{T}\left(t x_{i}\right) \geq t(1+\varepsilon / 4)$ for large $t$, implying that, for every $c \in(0,1)$, we have

$$
P\left(B\left(t x_{i}, \gamma\right) \subset S_{t(1+\varepsilon / 4)} \text { for some } i=1, \ldots, n\right) \leq c
$$

if $t$ is large. The idea of the proof is that, if $t A$ contains strongly infected points for large $t$, then with high probability some point $t x_{i}$ will be strongly infected within time $t \varepsilon / 4$ and this conflicts with (28). To formalize this intuition, let

$$
p=P\left(\tilde{S}_{t} \cap t A \neq \emptyset \text { for arbitrarily large } t\right)
$$


and assume for contradiction that $p>0$. For fixed $t$, write $E_{t}=\left\{t^{\prime} \geq t ; \tilde{S}_{t^{\prime}} \cap\right.$ $\left.t^{\prime} A \neq \emptyset\right\}$ and define

$$
T_{t}= \begin{cases}\inf E_{t} & \text { if } E_{t} \neq \emptyset \\ \infty & \text { if } E_{t}=\emptyset\end{cases}
$$

Note that $P\left(T_{t}<\infty\right) \geq p>0$ for each $t$. Consequently, we can condition on the event that $T_{t}<\infty$ and pick $y_{t}$ uniformly on $\tilde{S}_{T_{t}} \cap T_{t} A$. By Lemma 5.1,

$$
P\left(B\left(y_{t}, T_{t} \delta \varepsilon / 4\right) \subset S_{T_{t}(1+\varepsilon / 4)}^{\left(y_{t}, T_{t}\right)} \mid T_{t}<\infty\right) \geq 1-e^{-c t \varepsilon / 4},
$$

that is, the ball with radius $T_{t} \delta \varepsilon / 4$ around $y_{t}$ is with high probability infected at time $T_{t}(1+\varepsilon / 4)$ in a process started from $y_{t}$ at time $T_{t}$. If $t$ is large this implies that the ball with radius $T_{t} \delta \varepsilon / 8$ is strongly infected with high probability. Formally,

$$
P\left(B\left(y_{t}, T_{t} \delta \varepsilon / 8\right) \subset \tilde{S}_{T_{t}(1+\varepsilon / 4)}^{\left(y_{t}, T_{t}\right)} \mid T_{t}<\infty\right) \geq 1-e^{-c t \varepsilon / 4} .
$$

Since $y_{t}$ is strongly infected at time $T_{t}$ we obtain

$$
P\left(B\left(y_{t}, T_{t} \delta \varepsilon / 8\right) \subset \tilde{S}_{T_{t}(1+\varepsilon / 4)} \mid T_{t}<\infty\right) \rightarrow 1 \quad \text { as } t \rightarrow \infty .
$$

Now, by (27), $T_{t} A$ is covered by the balls $B\left(T_{t} x_{i}, T_{t} \delta \varepsilon / 8\right)$. Hence, since $y_{t} \in$ $T_{t} A$, we can find at least one point $x_{i}$ such that $T_{t} x_{i} \in B\left(y_{t}, T_{t} \delta \varepsilon / 8\right)$ and, consequently,

$$
P\left(B\left(T_{t} x_{i}, \gamma\right) \subset S_{T_{t}(1+\varepsilon / 4)} \text { for some } i=1, \ldots, n \mid T_{t}<\infty\right) \rightarrow 1 \quad \text { as } t \rightarrow \infty \text {. }
$$

Pick $t$ large so that the above probability is greater than $1 / 2$ and so that (28) holds for $c=p / 4$. Use the fact that $P\left(T_{t}<\infty\right) \geq p$ for each $t$ to obtain

$$
\begin{aligned}
& P\left(\exists t^{\prime} \geq t \text { such that } B\left(t^{\prime} x_{i}, \gamma\right) \subset S_{t^{\prime}(1+\varepsilon / 4)} \text { for some } i=1, \ldots, n\right) \\
\geq & P\left(B\left(T_{t} x_{i}, \gamma\right) \subset S_{T_{t}(1+\varepsilon / 4)} \text { for some } i=1, \ldots, n \mid T_{t}<\infty\right) P\left(T_{t}<\infty\right) \\
> & p / 2 .
\end{aligned}
$$

This contradicts (28). Hence we must have $p=0$, that is, almost surely $\tilde{S}_{t} \cap t A=$ $\emptyset$ for large $t$. It remains to show that (ii) follows from this. To this end, let $\Gamma$ denote the set of outbursts that occur in $t A$ for some $t$ during the progress of the growth, that is, $\Gamma$ is the set of outbursts $\left(X_{n}, T_{n}\right)$ such that $X_{n} \in T_{n} A$. Furthermore, let $\Gamma_{\gamma}$ be those outbursts in $\Gamma$ whose radius is at least $\gamma$. Assume that (ii) fails so that with positive probability $S_{t} \cap\left[(1+\varepsilon) t B\left(0, \mu^{-1}\right)\right]^{c} \neq \emptyset$ for arbitrarily large $t$. This implies that $P(|\Gamma|=\infty)>0$. Now, each time an outburst takes place we condition on the process up to that time, including the fact that an outburst takes place at that time at that location, but excluding the radius of the outburst. Since the radii of the outbursts are i.i.d. it follows from Levy's version of the Borel-Cantelli lemma (see Williams (1991), section 12.5) that we can not have $|\Gamma|=\infty$ and $\left|\Gamma_{\gamma}\right|<\infty$. Hence the fact that 
$P(|\Gamma|=\infty)>0$ implies that $P\left(\left|\Gamma_{\gamma}\right|=\infty\right)>0$ as well. But, if $\left|\Gamma_{\gamma}\right|=\infty$, then the region $t A$ must contain strongly infected points for arbitrarily large $t$, and hence we have derived a contradiction.

At this point, (i) and (ii) are established and all that remains is to note that, for $t>\max \left\{T^{1}, T^{2}\right\}$, we have

$$
(1-\varepsilon) t B\left(0, \mu^{-1}\right) \subset S_{t} \subset(1+\varepsilon) t B\left(0, \mu^{-1}\right) .
$$

The shape theorem is proved.

We end by proving Theorem 1.2, that is, by proving that the asymptotic shape is independent of the state at time zero.

Proof of Theorem 1.2: For an arbitrary set $\Pi \subset \mathbb{R}^{d}$, introduce generalized versions of the quantities $S_{t}$ and $S_{t}^{(x, s)}$ as follows: Let $S_{t}^{\Pi}$ denote the region infected at time $t$ in a process started from the set $\Pi$ and let $S_{t}^{(\Pi, s)}$ denote the region infected at time $t, t \geq s$, in a process started from the set $\Pi$ at time $s$. We will show that, for any $\varepsilon>0$, almost surely

$$
(1-\varepsilon) t B\left(0, \mu^{-1}\right) \subset \frac{S_{t}^{\Pi}}{t} \subset(1+\varepsilon) t B\left(0, \mu^{-1}\right)
$$

if $t$ is large. To this end, let $\tau$ be the first time when $\Pi$ is contained in $S_{t}$, and let $\tau^{\prime}$ be the first time counting from $\tau$ when $B(0, \gamma)$ is contained in $S_{t}^{(\Pi, \tau)}$, that is,

$$
\tau=\inf \left\{t ; \Pi \subset S_{t}\right\}
$$

and

$$
\tau^{\prime}=\inf \left\{t ; B(0, \gamma) \subset S_{\tau+t}^{(\Pi, \tau)}\right\} .
$$

If $\Pi$ is bounded and has strictly positive Lebesgue measure these times are both finite with probability 1 . Furthermore,

$$
S_{\tau+\tau^{\prime}+t}^{\left(B(0, \gamma), \tau+\tau^{\prime}\right)} \subset S_{\tau+\tau^{\prime}+t}^{(\Pi, \tau)} \subset S_{\tau+\tau^{\prime}+t}
$$

which implies that

$$
\frac{S_{\tau+\tau^{\prime}+t}^{\left(B(0, \tau), \tau^{\prime}\right)}}{t} \subset \frac{\tau^{\prime}+t}{t} \cdot \frac{S_{\tau+\tau^{\prime}+t}^{(\Pi, \tau)}}{\tau^{\prime}+t} \subset \frac{\tau+\tau^{\prime}+t}{t} \cdot \frac{S_{\tau+\tau^{\prime}+t}}{\tau+\tau^{\prime}+t} .
$$

The quotients $\left(\tau^{\prime}+t\right) / t$ and $\left(\tau+\tau^{\prime}+t\right) / t$ tend to 1 as $t \rightarrow \infty$ and by Theorem 1.1 the asymptotic shape for the processes $S_{\tau+\tau^{\prime}+t}^{\left(B(0, \gamma), \tau+\tau^{\prime}\right)} / t$ and $S_{\tau+\tau^{\prime}+t} /\left(\tau+\tau^{\prime}+t\right)$ is $B\left(0, \mu^{-1}\right)$. This establishes (29).

\section{Acknowledgement}

I thank my thesis advisor Olle Häggström for proposing the problem, for superb guidance during the progress of the work and for critical readings of the manuscript. I also thank Anders Martin-Löf for many stimulating discussions. Finally, I tnak an anonymous referee for his or her careful reading and constructive comments. 


\section{References}

Boivin, D. (1990): First passage percolation: the stationary case, Prob. Th. Rel. Fields 86, 491-499.

Bramson, M. and Griffeath, D. (1981): On the Williams-Bjerknes tumour growth model I, Ann. Prob. 9, 173-185.

Cox, J.T. and Durrett, R. (1981): Some limit theorems for percolation processes with necessary and sufficient conditions, Ann. Prob. 9, 583-603.

Durrett, R. (1988): Lecture Notes on Particle Systems and Percolation, Wadsworth \& Brooks/Cole.

Eden, M. (1961): A two-dimensional growth process, Proceedings of the 4th Berkeley symposium on mathematical statistics and probability vol. IV, 223239, University of California press.

Howard C.D. and Newman C.M. (1997): Euclidean models of first-passage percolation, Prob. Th. Rel. Fields 108, 153-170.

Kesten, H. (1986): Aspects of first-passage percolation, Lecture Notes in Mathematics, vol. 1180, 125-264, Springer .

Lee, T. and Cowan, R. (1994): A stochastic tessellation of digital space, Mathematical morphology and its applications to image processing, 217-224, Kluwer.

Liggett, T.M. (1985): An improved subadditive ergodic theorem, Ann. Prob. 13, 1279-1285.

Penrose, M. (2001): Random parking, sequential adsorption and the jamming limit, Commun. Math. Phys. 218, 153-176.

Richardson, D. (1973): Random growth in a tessellation, Proc. Cambridge Phil. Soc. 74, 515-528.

Williams, D. (1991): Probability with Martingales, Cambridge University Press.

Williams, T. and Bjerknes R. (1972): Stochastic model for abnormal clone spread through epithelial basal layer, Nature 236, 19-21. 